B. Fagerberg $\cdot$ S. Edwards $\cdot$ T. Halmos $\cdot$ J. Lopatynski $\cdot$ H. Schuster $\cdot$ S. Stender

G. Stoa-Birketvedt $\cdot$ S. Tonstad $\cdot$ S Halldórsdóttir · I. Gause-Nilsson

\title{
Tesaglitazar, a novel dual peroxisome proliferator-activated receptor $\alpha / Y$ agonist, dose-dependently improves the metabolic abnormalities associated with insulin resistance in a non-diabetic population
}

Received: 2 February 2005 / Accepted: 16 April 2005 / Published online: 7 July 2005

(C) Springer-Verlag 2005

\begin{abstract}
Aims/hypothesis: Insulin resistance is associated with abnormalities in lipid and glucose metabolism, which are major components of metabolic syndrome and risk factors for vascular disease. This study examined the effect of tesaglitazar (Galida), a novel, dual-acting peroxisome
\end{abstract}

B. Fagerberg $(\bowtie)$

Wallenberg Laboratory for Cardiovascular Research,

Sahlgrenska University Hospital,

41345 Göteborg, Sweden

e-mail: bjorn.fagerberg@wlab.gu.se

Tel.: +46-31-3421530

Fax: +46-31-823762

S. Edwards

The North Cardiff Medical Centre,

Cardiff, UK

T. Halmos

National Korányi Institution for Pulmonary Diseases,

Budapest, Hungary

J. Lopatynski

Department of Primary Health Services and Family Medicine,

Lublin Medical Academy,

Lublin, Poland

H. Schuster

Humboldt University,

Berlin, Germany

S. Stender

Gentofte University Hospital,

Copenhagen, Denmark

G. Stoa-Birketvedt

University Hospital,

Tromso, Norway

S. Tonstad

Ullevål University Hospital,

Oslo, Norway

S. Halldórsdóttir · I. Gause-Nilsson

AstraZeneca R\&D,

Mölndal, Sweden proliferator-activated receptor $\alpha / \gamma$ agonist, on lipid and glucose metabolism in patients with evidence of insulin resistance. Methods: A 12-week, multicentre, randomised, double-blind, placebo-controlled, dose-finding study compared the efficacy and safety of oral tesaglitazar $(0.1,0.25$, 0.5 and $1.0 \mathrm{mg} /$ day) and placebo in 390 non-diabetic patients with hypertriglyceridaemia (plasma triglyceride concentration $>1.7 \mathrm{mmol} / \mathrm{l}$ ) and abdominal obesity (waistto-hip ratio $>0.90$ for men and $>0.85$ for women). Results: A 1.0-mg dose of tesaglitazar reduced fasting triglycerides (the primary endpoint) by $37 \%(95 \% \mathrm{CI}$ : $-43 \%$ to $-30 \% ; p<0.0001$ ), non-HDL-cholesterol by $15 \%$ (95\% CI: $-20 \%$ to $-10 \% ; p<0.0001)$ and NEFA by $40 \%$ (95\% CI: $-51 \%$ to $-27 \% ; p<0.0001$ ), and increased HDLcholesterol by $16 \%$ (95\% CI: 8 to $-24 \% ; p<0.0001)$. At the end of treatment there was a dose-dependent increase in patients with pattern A LDL particle diameter $(40 \%$ at baseline vs $87 \%$ at 12 weeks for tesaglitazar $1.0 \mathrm{mg}$ ). Tesaglitazar produced significant reductions in fasting insulin concentration $(-35 \% ; p<0.0001)$ and plasma glucose concentration $(-0.47 \mathrm{mmol} / 1 ; p<0.0001)$. Respiratory infection and gastrointestinal symptoms were the most common adverse events and were similarly frequent in all groups. Conclusions/interpretation: Tesaglitazar was well tolerated and produced significant, dose-dependent improvements in lipid and glucose metabolism and insulin sensitivity. Tesaglitazar may have the potential to prevent vascular complications and delay progression to diabetes in these patients.

Keywords Cardiovascular risk factors - Dyslipidaemia . HDL-cholesterol · Hyperinsulinaemia · Hypoglycaemic agents $\cdot$ Insulin resistance $\cdot$ LDL-cholesterol - Peroxisome proliferator-activated receptor $\cdot$ Triglycerides

Abbreviations FPG: fasting plasma glucose $\cdot$ HOMA: homeostasis model assessment - ITT: intent-to-treat (population) - NMR: nuclear magnetic resonance - PPAR: peroxisome proliferator-activated receptor - SIR: Study in Insulin Resistance 


\section{Introduction}

Insulin resistance is a key underlying factor in a number of metabolic abnormalities, including type 2 diabetes and metabolic syndrome $[1,2]$. Metabolic syndrome comprises a constellation of abnormalities, including abdominal obesity, high blood pressure, high triglycerides, low HDL-cholesterol and high fasting plasma glucose (FPG). Typically, patients with metabolic syndrome are insulinresistant, have a highly atherogenic dyslipidaemic profile [3], and most will eventually progress to type 2 diabetes [4-6].

It is now recognised that insulin resistance and atherogenic dyslipidaemia may exist and remain undiagnosed for a number of years. Although some patients with metabolic syndrome have a near-normal glucose profile, they are at significantly increased risk of cardiovascular disease compared with individuals without metabolic syndrome. In the Finnish Botnia study, the risk of coronary heart disease was increased 3-fold in patients with the metabolic syndrome [7], and a US study reported a 2.4- and 5.9-fold greater risk of coronary heart disease in men and women, respectively, with clusters of three or more metabolic risk factors [8]. Among men with the metabolic syndrome, data from the West of Scotland Coronary Prevention Study indicated a 3.7-fold increase in risk of coronary heart disease [9], and the Kuopio Ischaemic Heart Disease Risk Factor Study reported a 2.9- to 4.2-fold increase in the risk of cardiovascular mortality [10]. Among women with a history of coronary heart disease, those with metabolic syndrome were significantly more likely to experience myocardial infarction, stroke or coronary death [11]. Without intervention, these individuals usually have advanced atherosclerosis by the time diabetes is diagnosed.

At one time, diabetes mellitus was thought to be characterised solely by impaired glucose metabolism. Although the diagnosis of diabetes is still defined by fasting hyperglycaemia and/or blood glucose concentrations $2 \mathrm{~h}$ after an oral glucose load, type 2 diabetes is increasingly seen as a complex metabolic disorder in which abnormalities of lipid metabolism precede the appearance of hyperglycaemia [12]. The typical dyslipidaemia of type 2 diabetes comprises hypertriglyceridaemia, elevation of plasma NEFA, altered LDL-cholesterol profiles and decreased HDL-cholesterol [13].

Tesaglitazar (Galida) is a novel, dual-acting agonist of peroxisome proliferator-activated receptor $\alpha / \gamma(\operatorname{PPAR} \alpha / \gamma)$ that has been shown to improve insulin resistance and both glucose and lipid abnormalities in rodent models of insulin resistance and diabetes [14]. Preliminary studies in volunteers have shown that tesaglitazar is rapidly and completely absorbed after oral dosing and has linear pharmacokinetic properties, with an elimination half-life of between 38 and $59 \mathrm{~h} \mathrm{[15].}$

Our dose-finding study, the Study in Insulin Resistance (SIR), examined the effect of tesaglitazar given once daily on both lipid and glucose metabolism in individuals with hypertriglyceridaemia and abdominal obesity, two of the risk factors associated with the metabolic syndrome [16, 17]. Studies have shown that the majority of such individuals are insulin resistant and are likely to develop type 2 diabetes [18-20]. The primary endpoint was the change in serum triglyceride after 12 weeks of treatment. Secondary endpoints included changes in total cholesterol, HDL-cholesterol and LDL-cholesterol, LDL particle size and concentration, NEFA, insulin, glucose and $\mathrm{HbA}_{1} \mathrm{c}$.

\section{Subjects, materials and methods}

\section{Ethical considerations}

This study (SH-SBT-0001) was designed and carried out according to the Declaration of Helsinki (version amended October 2000) and local guidelines in participating countries. Appropriate institutional review boards approved the research protocol and consent forms, and all patients gave their written, informed consent before initiation of any trial procedure.

\section{Patients}

This was a multicentre, randomised, double-blind, placebo-controlled, five-arm parallel-group, dose-finding study (Fig. 1). Patients were outpatient men and women (either

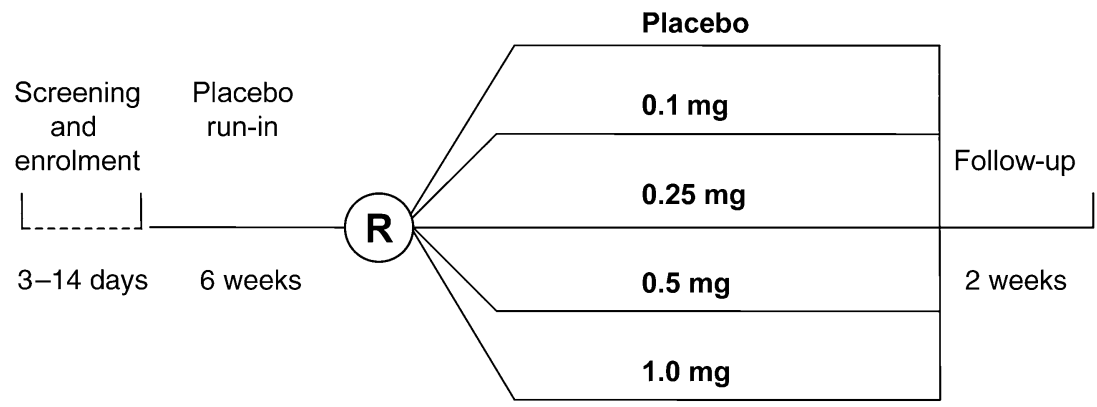


surgically sterile or who had their last menstruation more than 12 months previously) over 30 years of age. All patients had fasting serum triglyceride concentrations of $1.7-$ $7.0 \mathrm{mmol} / \mathrm{l}(150-620 \mathrm{mg} / \mathrm{dl})$ that varied by no more than $40 \%$ when tested 10 and 3 days before randomisation, and waist-to-hip ratios $>0.90$ for men and $>0.85$ for women.

Patients were enrolled at a screening visit 7-9 weeks before randomisation. Patients were excluded if they had either fasting serum total cholesterol $>8.0 \mathrm{mmol} / 1$ (309 mg/ dl) or serum creatinine $>120 \mu \mathrm{mol} / \mathrm{l}$. Patients with a history of diabetes were excluded if they had FPG concentrations $>7.0 \mathrm{mmol} / \mathrm{l}(126 \mathrm{mg} / \mathrm{dl})$ and if they had used insulin or oral antidiabetic drugs within the 12 weeks before enrolment. Patients were also excluded if they met any of the following criteria: systolic blood pressure $\geq 170 \mathrm{mmHg}$ and/or diastolic blood pressure $\geq 100 \mathrm{mmHg}$; BMI $>40$; history of ischaemic heart disease, asthma, liver disease, endocrine disorder (except for controlled primary hypothyroidism or isolated elevation of serum thyroid-stimulating hormone up to 1.5 times the upper reference limit), pancreatitis, cancer, gout or psychiatric disorder; current major disease as judged relevant by the investigator; concomitant use of lipid-lowering drugs and other drugs that could affect efficacy variables; use of insulin or oral antidiabetic drugs within the 12 weeks before enrolment; alcohol or other substance abuse; or participation in a clinical trial evaluating an investigational drug or blood donation in the preceding 12 weeks. Patients were also excluded from the study if on the day of randomisation they had an FPG concentration of $>7.0 \mathrm{mmol} / \mathrm{l}(126 \mathrm{mg} / \mathrm{dl})$. Patient enrolment began in June 2000 and the last patient completed the study in May 2001.

\section{Treatments}

At enrolment, patients were given standardised advice about diet and lifestyle (smoking, alcohol consumption and physical activity) applicable to this group of individuals. Patients began a 6-week, single-blind run-in period during which they took one placebo tablet each morning. At the end of this period, patients were randomised to one of five treatments: placebo or $0.1,0.25,0.5$ or $1.0 \mathrm{mg}$ of tesaglitazar, taken as a single tablet with water once each morning after an overnight fast. Patients continued to take the treatment for up to 86 days, after which they entered a 14-day follow-up period during which they took neither placebo nor tesaglitazar.

\section{Efficacy measures}

Patients had assessments after an overnight fast, and at each visit waist and hip circumference and weight were recorded. Blood samples were taken (before breakfast and before patients took the study medication) and the following were determined: serum triglyceride, total cholesterol and HDL-cholesterol, and plasma LDL-cholesterol, LDL particle size and concentration, insulin, glucose and
$\mathrm{HbA}_{1} \mathrm{c}$. In addition, fasting plasma NEFA was measured in a subpopulation of 176 patients.

Primary efficacy was compared between baseline and after 12 weeks of treatment; another comparison was made at the end of the final 2-week washout period. The primary efficacy variable was change in fasting serum triglyceride from baseline to the end of the 12-week treatment period.

All variables except LDL concentration and particle size were measured using standard laboratory procedures at the central laboratory of each study centre. LDL concentration and particle size were calculated by nuclear magnetic resonance (NMR) spectroscopy as previously described [21].

\section{Derived variables}

Non-HDL-cholesterol was calculated as total cholesterol minus HDL-cholesterol and the triglyceride : HDL-cholesterol ratio was determined. Insulin resistance was calculated using (1) the homeostasis model assessment: HOMA= $\mathrm{FPG} \times \mathrm{PI} \div 22.5$, where $\mathrm{FPG}$ is in $\mathrm{mmol} / \mathrm{l}$ and $\mathrm{PI}$ is plasma insulin concentration in $\mathrm{mIU} / \mathrm{l}$. (Since insulin was measured in pmol/1, a correction factor of $1 / 7$ was applied, hence HOMA=FPG $\times \mathrm{PI} \div 157.5$ ) [22]; and (2) the product of fasting plasma insulin and fasting plasma NEFA (measured in the NEFA subpopulation of patients) [22].

Patients were classified as either LDL pattern A or pattern $\mathrm{B}$ according to the distribution of lipoprotein particle sizes. Patients with an average LDL particle size $>20.5 \mathrm{~nm}$ were classified as pattern $\mathrm{A}$, and patients with an average LDL particle size $\leq 20.5 \mathrm{~nm}$ were classified as pattern B. VLDL particle size was also determined using the NMR procedure.

\section{Safety}

Adverse events were recorded at each visit and were classified according to a standardised dictionary derived from the World Health Organization's Adverse Reactions Terminology dictionary [23]. Venous blood samples were drawn before breakfast and intake of study medication, and after $3 \mathrm{~min}$ of supine rest at 2- to 4-week intervals throughout the study period. Laboratory values assessed from blood samples in the safety screening included erythrocyte sedimentation rate, counts of white blood cells, differential white blood cells, platelets, red blood cells, and reticulocytes, haemoglobin concentration, mean cell haemoglobin, and mean cell haemoglobin concentration. In addition, serum aspartate aminotransferase, alanine aminotransferase, $\gamma$-glutamyl transferase, alkaline phosphatase, albumin, total bilirubin, creatinine, haptoglobin, $\mathrm{Na}^{+}, \mathrm{K}^{+}, \mathrm{Ca}^{2+}$, free serum thyroxine and serum thyroid-stimulating hormone were measured.

Standard physical examinations were carried out and resting ECGs recorded before the run-in period and again at the end of treatment. Heart rate and blood pressure were measured after 5 min supine rest, both before and on four 
occasions during the randomised phase of the study. Urine samples for dipstick analysis of albumin, glucose and haemoglobin were collected in the morning at 2- to 4-week intervals throughout the study period.

\section{Statistical methods}

Statistical analyses of efficacy were based on the intent-totreat (ITT) population. The ITT population included all patients who took at least one dose of drug during the double-blind treatment phase and who had some efficacy data both at baseline and after randomisation. Missing data at the end of the treatment period were imputed using the last observation carried forward principle.

All efficacy variables except plasma glucose and $\mathrm{HbA}_{1} \mathrm{c}$ were log-transformed before statistical testing; means are therefore geometric. The change in fasting serum triglycerides was analysed in a linear model using a fixed-effect analysis of covariance with treatment and country as factors and the baseline value as covariate. Confidence intervals are two-sided and Dunnett's method was used to maintain a simultaneous confidence level of 5\%. The other efficacy variables were analysed in a similar manner. Safety variables are presented using descriptive and summary statistics.

To detect a difference of $15 \%$ in mean fasting serum triglyceride between the tesaglitazar and placebo groups from baseline to week 12, 50 patients were required to complete each active treatment arm and 100 were required to complete the placebo arm. The calculation was based on a residual between-patient standard deviation of 0.24 for the change from baseline in log serum triglycerides. The significance level was set at a conservative 0.0125 to allow for a Bonferroni correction for four primary comparisons.

\section{Results}

Of the 656 patients who were enrolled, 397 met the entry criteria and were randomised into the study (Fig. 2). Seven patients were not included in the ITT analysis because of lack of efficacy data or because they received no study drug during the double-blind period. After randomisation, 47 patients were withdrawn: most were withdrawn by investigators because of delays in the processing of blood samples - patients were randomised without final laboratory results or were later found to have violated the inclusion/ exclusion criteria before randomisation. Ten patients withdrew their consent. Similar numbers of patients were withdrawn from each study group, and it is not likely that the withdrawals affected the analysis. Although they did not complete the study, these patients were included in the ITT analysis.

\section{Demographics}

Baseline characteristics of the patients were not significantly different between the treatment groups (Table 1).
Fig. 2 Flow chart of study of insulin resistance. ${ }^{*}$ Note that a patient could have more than one reason for being excluded from analysis

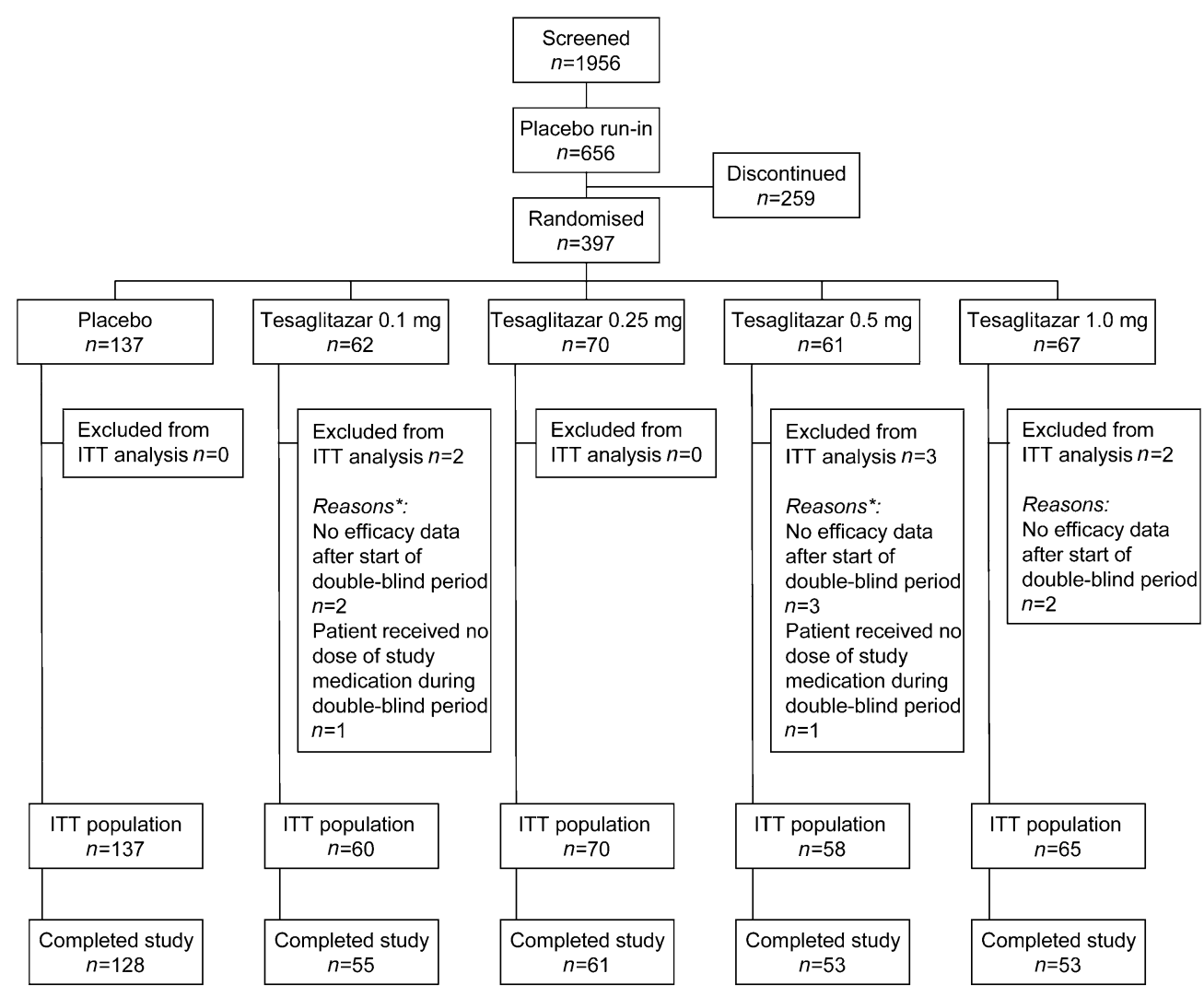


Table 1 Baseline characteristics of patients (ITT population)

Values are means (SD)

\begin{tabular}{lllllll}
\hline & Placebo & \multicolumn{2}{l}{ Tesaglitazar } & \multicolumn{2}{c}{ Total } \\
\cline { 3 - 6 } & & $0.1 \mathrm{mg}$ & $0.25 \mathrm{mg}$ & $0.5 \mathrm{mg}$ & $1.0 \mathrm{mg}$ & \\
\hline$n$ & 137 & 60 & 70 & 58 & 65 & 390 \\
Age (years) & $50(11)$ & $52(9)$ & $49(10)$ & $51(10)$ & $51(11)$ & $50(10)$ \\
Men/women & $107 / 30$ & $43 / 17$ & $60 / 10$ & $43 / 15$ & $49 / 16$ & $302 / 88$ \\
Previous diabetes diagnosis $(n)$ & 1 & 1 & 1 & 0 & 0 & 3 \\
History of hypertension (\%) & 16 & 17 & 14 & 21 & 22 & 17 \\
BMI (kg m $\left.{ }^{2}\right)$ & $31(4)$ & $31(4)$ & $30(4)$ & $31(4)$ & $31(4)$ & $31(4)$ \\
Waist-to-hip ratio & $0.98(0.07)$ & $0.97(0.07)$ & $0.98(0.06)$ & $0.97(0.06)$ & $0.98(0.08)$ & $0.98(0.07)$ \\
Serum triglyceride & & & & & & \\
$(\mathrm{mmol} / \mathrm{l})$ & $3.0(1.0)$ & $3.2(1.1)$ & $2.9(1.0)$ & $2.8(0.7)$ & $2.9(0.9)$ & $3.0(0.9)$ \\
$(\mathrm{mg} / \mathrm{dl})$ & $270(88)$ & $280(94)$ & $252(84)$ & $247(65)$ & $259(78)$ & $270(80)$ \\
Plasma insulin (pmol/1) & $95(68)$ & $88(40)$ & $88(50)$ & $110(69)$ & $91(49)$ & $95(59)$ \\
\hline
\end{tabular}

Over three-quarters of patients (77\%) were male. Baseline characteristics were similar for men and women, except that the women were older ( 57 years vs 48 years for men).

Primary efficacy variable

Tesaglitazar produced significant dose-dependent reductions in fasting serum triglyceride (Fig. 3). Placebo-corrected reductions for the $0.25,0.5$ and $1.0 \mathrm{mg}$ doses of tesaglitazar were $-16 \%(95 \% \mathrm{CI}:-24 \%$ to $-7 \% ; p<0.0001)$, $-27 \%(95 \%$ CI: $-35 \%$ to $-19 \% ; p<0.0001)$ and $-37 \%$ (95\% CI: $-43 \%$ to $-30 \% ; p<0.0001$ ), respectively. Serum triglyceride concentrations declined over time up to 4 weeks, after which they remained stable until active drug intake was stopped at 12 weeks. During the subsequent 2-week, drug-free follow-up, serum triglyceride concentrations increased towards baseline (data not shown).

Secondary efficacy variables: lipids

The two highest doses of tesaglitazar significantly increased serum HDL-cholesterol. Placebo-corrected increases of $11 \%(95 \%$ CI: $3-19 \% ; p=0.003)$ and $16 \%$
(95\% CI: 8-24\%; $p<0.0001$ ) were seen in the 0.5 - and $1.0-$ mg groups, respectively (Fig. 3). The triglyceride/HDL ratio was also significantly reduced at all doses of tesaglitazar. The greatest placebo-corrected reduction (48\%) was seen with the 1.0-mg dose of tesaglitazar (Table 2). Serum non-HDL-cholesterol was significantly reduced in the two highest tesaglitazar dose groups (Fig. 3). Tesaglitazar also reduced serum total cholesterol, although only the highest dose produced a statistically significant fall $(8 \%, p<0.0001)$ (Table 2). In addition, the ratio of plasma LDL-cholesterol/ plasma HDL-cholesterol fell in response to tesaglitazar (Table 2).

A significant dose-dependent effect was seen in the subpopulation in which fasting plasma NEFA was measured: tesaglitazar $1.0 \mathrm{mg}$ produced a placebo-corrected reduction of $40 \%(p<0.0001)$ in NEFA concentration after 12 weeks of treatment. Significant reductions were also seen with the $0.25-\mathrm{mg}(-18 \%, p=0.05)$ and $0.5-\mathrm{mg}(-21 \%$, $p=0.01$ ) doses (Table 2).

There was a significant increase in the diameter of plasma LDL particles in patients treated with tesaglitazar. When patients were stratified according to the mean size of their LDL particles, a dose-dependent increase in the number of patients with the less atherogenic pattern A (mean diameter of LDL particles $>20.5 \mathrm{~nm}$ ) from approximately
Fig. 3 Effect of tesaglitazar on fasting serum triglycerides, serum HDL-cholesterol and serum total cholesterol (ITT population). Placebo-corrected changes from baseline with $95 \%$ CIs. White bars, tesaglitazar $0.10 \mathrm{mg}$; striped bars, tesaglitazar $0.25 \mathrm{mg}$; grey bars, tesaglitazar $0.50 \mathrm{mg}$; dotted bars, tesaglitazar $1.00 \mathrm{mg}$ ${ }^{*} p<0.05$; ${ }^{*} p<0.0001 ; T G=$ triglycerides; $H D L-C=$ high-density lipoprotein cholesterol

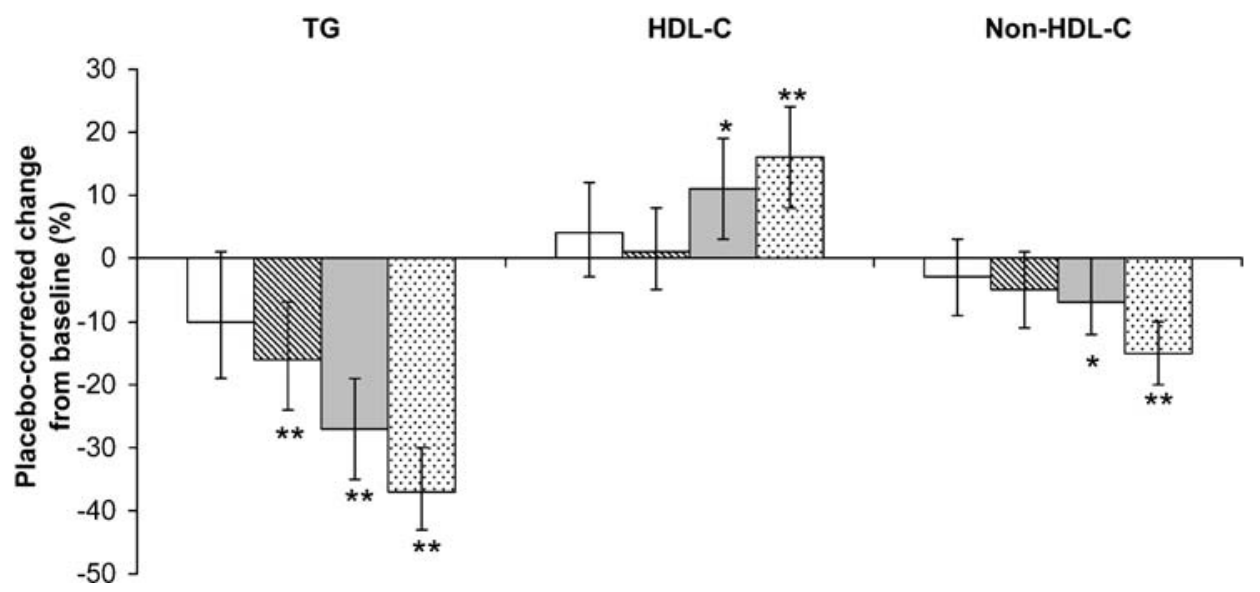


$40 \%$ at baseline to $87 \%$ after 12 weeks of treatment with tesaglitazar $1.0 \mathrm{mg}$ (Fig. 4) was found. Of the patients receiving the 1.0-mg dose of tesaglitazar and who had pattern B at start of treatment $(n=38), 30(\sim 79 \%)$ switched to pattern $A$ at the end of treatment. None of the patients in the tesaglitazar 1.0-mg group switched from pattern A at baseline to pattern $\mathrm{B}$ at the end of treatment. In comparison, among patients on placebo who were pattern $\mathrm{B}$ at baseline $(n=82), 16(\sim 20 \%)$ switched to pattern A and 25\% (13/53) of those who were pattern $\mathrm{A}$ at baseline switched to pattern B.

Tesaglitazar also reduced plasma VLDL-cholesterol concentration and VLDL particle size in a dose-dependent manner (Table 2). At 12 weeks, treatment with $1.0 \mathrm{mg}$ tesaglitazar reduced VLDL-cholesterol concentration by $46 \%(p<0.0001)$ and mean VLDL particle size by $15 \%$ $(p<0.0001)$.

Secondary efficacy variables: insulin and glucose

All doses of tesaglitazar, except the lowest, significantly reduced fasting insulin at 12 weeks. Tesaglitazar also produced significant reductions in the HOMA index at all except the lowest dose tested (Fig. 5). Another useful index of insulin resistance is the product of fasting plasma insulin and fasting plasma NEFA. This index was assessed in a subpopulation of 164 patients, in whom tesaglitazar produced marked reductions at all doses except $0.1 \mathrm{mg}$ (Table 2).

Tesaglitazar had a clinically relevant effect on glucose metabolism. Although patients had baseline plasma glucose concentrations within the accepted normal reference range, tesaglitazar significantly reduced FPG at all doses except the lowest (Table 2). There were no statistically significant changes in $\mathrm{HbA}_{1} \mathrm{c}$ measurements in any of the study groups. Values at the end of treatment ranged from $5.81 \%$ to $5.90 \%$.

\section{Body weight (data not shown)}

The two highest doses of tesaglitazar $(0.5$ and $1.0 \mathrm{mg})$ produced small but significant increases in body weight: placebo-corrected increases were $0.90 \mathrm{~kg}(p=0.03)$ and $0.96 \mathrm{~kg}(p=0.01)$, respectively. There were no changes in waist-to-hip circumference ratios.

Table 2 Effect of tesaglitazar on secondary efficacy variables (ITT population)

\begin{tabular}{|c|c|c|c|c|c|c|c|c|}
\hline Variable & \multicolumn{2}{|l|}{$0.1 \mathrm{mg}$} & \multicolumn{2}{|l|}{$0.25 \mathrm{mg}$} & \multicolumn{2}{|l|}{$0.5 \mathrm{mg}$} & \multicolumn{2}{|l|}{$1.0 \mathrm{mg}$} \\
\hline NEFA $(\mathrm{mmol} / \mathrm{l})$ & $\begin{array}{l}0.52 \\
(0.14)\end{array}$ & $-17(-32$ to 1$)$ & $\begin{array}{l}0.53 \\
(0.22)\end{array}$ & $-18(-33$ to 0$)$ & $\begin{array}{l}0.48 \\
(0.17)\end{array}$ & $\begin{array}{l}-21(-35 \\
\text { to }-4)\end{array}$ & $\begin{array}{l}0.50 \\
(0.19)\end{array}$ & $\begin{array}{l}-40 \\
(-51 \text { to }-27)\end{array}$ \\
\hline $\begin{array}{l}\text { LDL-cholesterol/ } \\
\text { HDL-cholesterol }\end{array}$ & $\begin{array}{l}3.34 \\
(0.98)\end{array}$ & $0(-10$ to 10$)$ & $\begin{array}{l}3.90 \\
(1.17)\end{array}$ & $-7(-15$ to 3$)$ & $\begin{array}{l}3.77 \\
(0.99)\end{array}$ & $-5(-14$ to 5$)$ & $\begin{array}{l}3.66 \\
(0.99)\end{array}$ & $-11(-19$ to -2$)$ \\
\hline TG/HDL-cholesterol & $\begin{array}{l}2.96 \\
(1.44)\end{array}$ & $-15(-27$ to -1$)$ & $\begin{array}{l}2.63 \\
(1.40)\end{array}$ & $\begin{array}{l}-19 \\
(-30 \text { to }-6)\end{array}$ & $\begin{array}{l}2.49 \\
(0.98)\end{array}$ & $\begin{array}{l}-37 \\
(-46 \text { to }-26)\end{array}$ & $\begin{array}{l}3.01 \\
(1.53)\end{array}$ & $\begin{array}{l}-48 \\
(-55 \text { to }-39)\end{array}$ \\
\hline $\begin{array}{l}(\mathrm{mg} / \mathrm{dl}) \\
\text { VLDL-particle } \\
\text { diameter }(\mathrm{nm})\end{array}$ & $\begin{array}{l}215(81) \\
63(13)\end{array}$ & $-3(-9$ to 4$)$ & $\begin{array}{l}191(79) \\
59(12)\end{array}$ & $-7(-13$ to -1$)$ & $\begin{array}{l}180(72) \\
59(11)\end{array}$ & $\begin{array}{l}-12 \\
(-18 \text { to }-6)\end{array}$ & $\begin{array}{l}198(72) \\
60(11)\end{array}$ & $\begin{array}{l}-15 \\
(-21 \text { to }-9)\end{array}$ \\
\hline $\begin{array}{l}\text { (Fasting insulin)* } \\
\text { (fasting NEFA) } \\
\left(\mathrm{mmol}^{*} \mathrm{pmol} / \mathrm{l}^{2}\right)\end{array}$ & $44(22)$ & $-24(-42$ to 1$)$ & $49(37)$ & $-31(-48$ to -8$)$ & $59(46)$ & $\begin{array}{l}-34 \\
(-50 \text { to }-12)\end{array}$ & 48 (29) & $\begin{array}{l}-62 \\
(-72 \text { to }-50)\end{array}$ \\
\hline $\begin{array}{l}\text { Fasting plasma } \\
\text { glucose }\end{array}$ & & & & & & & & \\
\hline$(\mathrm{mmol} / \mathrm{l})$ & $\begin{array}{l}5.75 \\
(0.55)\end{array}$ & $\begin{array}{l}0.01 \\
(-0.15 \text { to } 0.17)^{\mathrm{a}}\end{array}$ & $\begin{array}{l}5.74 \\
(0.56)\end{array}$ & $\begin{array}{l}-0.20 \\
(-0.35 \text { to }-0.05)^{\mathrm{a}}\end{array}$ & $\begin{array}{l}5.65 \\
(0.53)\end{array}$ & $\begin{array}{l}-0.22 \\
(-0.38 \text { to }-0.06)^{\mathrm{a}}\end{array}$ & $\begin{array}{l}5.71 \\
(0.58)\end{array}$ & $\begin{array}{l}-0.47 \\
(-0.63 \text { to }-0.31)^{\mathrm{a}}\end{array}$ \\
\hline
\end{tabular}

Values are baseline means (SD) and placebo-corrected changes from baseline to 12 weeks $(95 \%$ confidence interval)

${ }^{\mathrm{a}}$ Glucose changes from baseline are expressed in $\mathrm{mmol} / \mathrm{l}(\mathrm{mg} / \mathrm{dl})$ 
Fig. 4 Distribution of plasma LDL-cholesterol particles after 12 weeks' treatment with tesaglitazar, intent-to-treat population. Grey bars, pattern A $>20.5 \mathrm{~nm}$, less atherogenic; black bars, pattern $\mathrm{B} \leq 20.5 \mathrm{~nm}$, more atherogenic

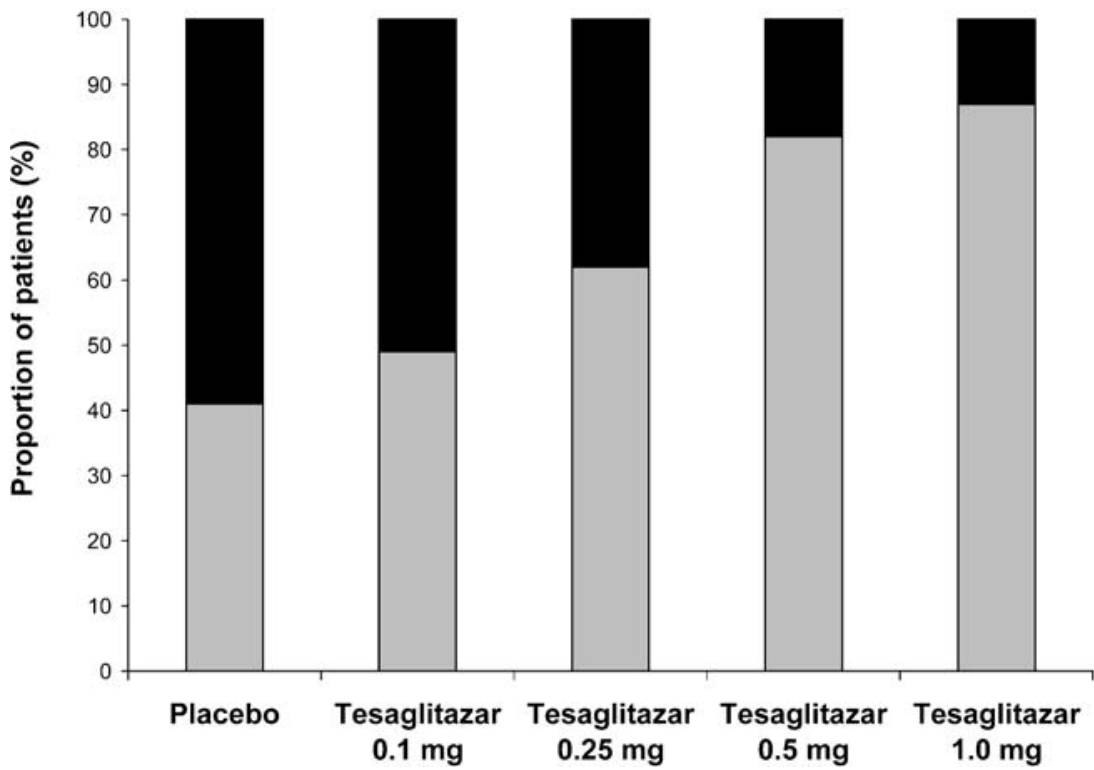

Safety

The safety population comprised all patients who took at least one dose of the drug during the double-blind treatment period and for whom subsequent study information was available. Overall, tesaglitazar was well tolerated by this population. Two patients experienced severe adverse events that were unrelated to the study medication: one in the placebo group was hospitalised for severe mental depression and one patient in the tesaglitazar 0.1-mg group suffered a ruptured Achilles tendon. Six patients withdrew from the study because of adverse events: one in the placebo group (iron and vitamin $\mathrm{B}_{12}$ deficiency, elevated homocysteine), two in the tesaglitazar $1.0-\mathrm{mg}$ group (stomach pains and nausea, respectively) and one in each of the other tesaglitazar dosage groups $(0.25 \mathrm{mg}$, itching dermatosis; $0.5 \mathrm{mg}$, bronchitis). In general, patients falling into these adverse-event categories were evenly distributed among the treatment groups, with no greater frequency in the tesaglitazar groups than in the placebo group (Table 3).

The most frequently reported adverse event was respiratory infection, reported by $16 \%$ of the placebo group and $12 \%, 7 \%, 16 \%$ and $11 \%$ of the tesaglitazar $0.1-, 0.25-, 0.5-$ and 1.0-mg groups, respectively. Gastrointestinal symptoms were also commonly reported, but the frequency of such symptoms was no higher in the active drug groups than in the placebo group. Haematological adverse events, such as anaemia, eosinophilia and thrombocytopaenia, were uncommon and equally distributed across the placebo and tesaglitazar groups.

Of the haematological laboratory values measured, haemoglobin concentration showed small decreases in patients randomised to tesaglitazar, ranging from a mean (SD) of $0.16(0.39) \mathrm{mmol} / \mathrm{l}$ in the $0.1-\mathrm{mg}$ group to $0.55(0.46)$
Fig. 5 Effect of tesaglitazar on fasting plasma insulin concentration and HOMA index, ITT population. Placebo-corrected changes from baseline with $95 \%$ CIs. White bars, tesaglitazar $0.10 \mathrm{mg}$; striped bars, tesaglitazar $0.25 \mathrm{mg}$; grey bars, tesaglitazar $0.50 \mathrm{mg}$; dotted bars, tesaglitazar $1.00 \mathrm{mg}$. ${ }^{*} p=0.05 ;{ }^{*} p \leq 0.01$; $* * * p<0.0001$. HOMA=homeostasis model assessmen

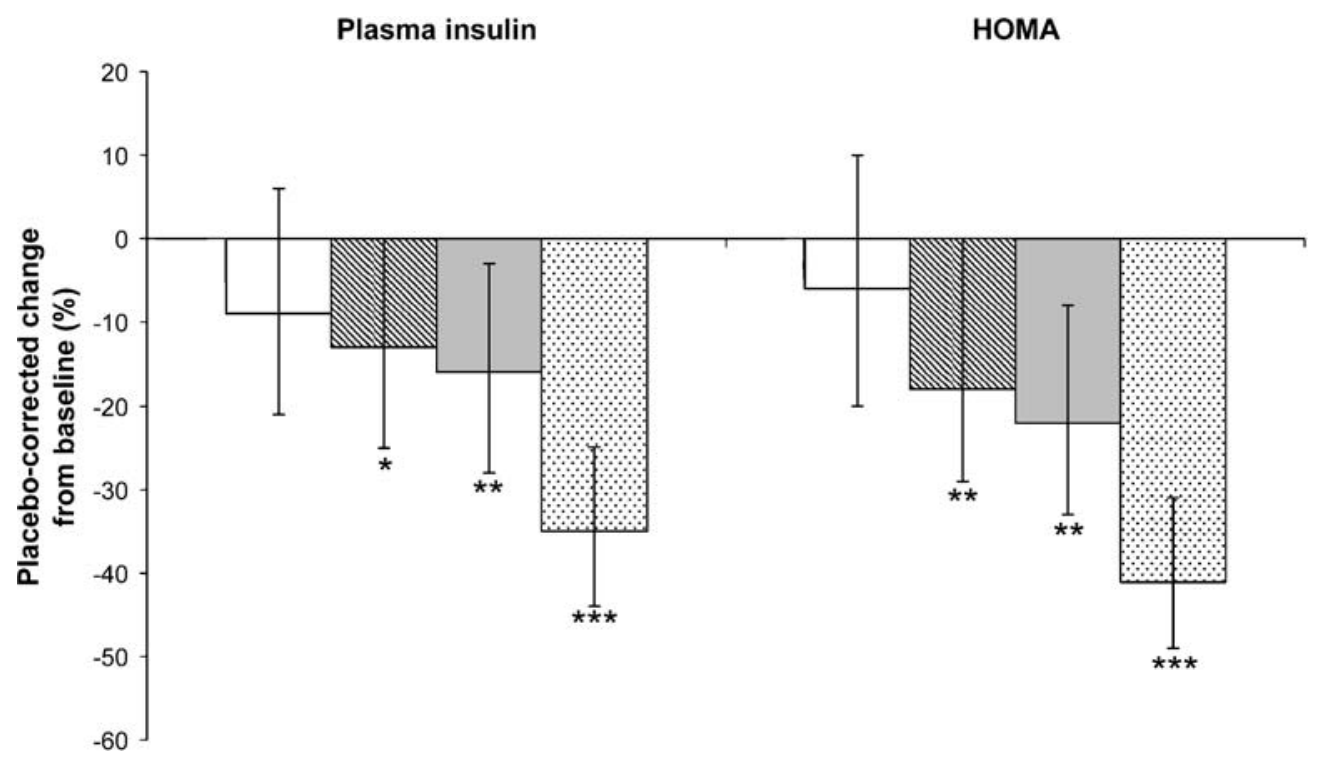


Table 3 Summary of adverse event (AE) categories during 12 weeks of treatment with tesaglitazar (ITT population)

\begin{tabular}{lccllc}
\hline & Placebo & \multicolumn{2}{l}{ Tesaglitazar } & & \\
\cline { 3 - 6 } & & $0.1 \mathrm{mg}$ & $0.25 \mathrm{mg}$ & $0.5 \mathrm{mg}$ & $1.0 \mathrm{mg}$ \\
\hline$n$ & 137 & 60 & 70 & 58 & 65 \\
Number (\%) of patients with & & & & & \\
Any AE & $75(54.7)$ & $39(65.0)$ & $36(51.4)$ & $39(67.2)$ & $39(60.0)$ \\
Fatal serious AE & 0 & 0 & 0 & 0 & 0 \\
Non-fatal serious AE & $1(0.7)$ & $1(1.7)$ & 0 & 0 & 0 \\
Drug stopped due to AE & $1(0.7)$ & $1(1.7)$ & $1(1.4)$ & $1(1.7)$ & $2(3.1)$ \\
Dosage reduced due to AE & 0 & 0 & 0 & 0 & 0 \\
Total number of AEs recorded & 178 & 78 & 73 & 89 & 64 \\
\hline
\end{tabular}

$\mathrm{mmol} / \mathrm{l}$ in the 1.0-mg group. This was accompanied by small dose-dependent reductions in haematocrit, ranging from $0.006(0.020)$ in the $0.1-\mathrm{mg}$ group to $0.021(0.025)$ in the $1.0-\mathrm{mg}$ group. Occasionally, patients were found to have small reductions in their leukocyte counts. A total of 15 of $249(6.0 \%)$ patients in the four tesaglitazar groups with normal leukocyte counts at baseline had a leukocyte count below the lower limit of normal $\left(3.8 \times 10^{9} / 1\right)$ at some time during the 12-week dosing period, compared with 7 of $134(5.2 \%)$ in the placebo group. There was no change in mean platelet count and no clinically relevant difference between platelet counts in the different treatment groups. There was no evidence that tesaglitazar caused any impairment of liver function.

During the first month of the study after randomisation, dose-dependent increases in mean serum creatinine concentrations were seen in patients randomised to tesaglitazar, ranging from a mean (SD) of 1 (9) $\mu \mathrm{mol} / 1$ in the 0.25 -mg group to $8(13) \mu \mathrm{mol} / \mathrm{l}$ in the $1.0-\mathrm{mg}$ group. After the first month, these changes stabilised and remained constant for the remainder of the study period. The values had all returned to normal by the end of the 2-week washout period at the end of the study. There was no evidence in this study that tesaglitazar caused proteinuria: U-albumin was unchanged.

There were no clinically relevant changes in heart rate or pulse in any of the treatment groups during the study. Although there were no clinically relevant changes in blood pressure during the study, there was a trend towards a reduction in the tesaglitazar 1.0-mg group (systolic reduction [SD]: 2 [15] mmHg; diastolic reduction: 1 [9] $\mathrm{mmHg}$ ). Isolated cases of oedema (one case in the placebo group and one in each of the three lowest tesaglitazar dosage groups) and left ventricular hypertrophy were reported (one in each of the lowest two tesaglitazar dosage groups), but there were no cases of cardiac failure. None of these adverse events were considered by investigators to be due to the study medication.

\section{Discussion}

These results show that tesaglitazar had significant dosedependent beneficial effects on the primary variable of fasting serum triglycerides in an insulin-resistant, nondiabetic population. Tesaglitazar also improved a range of lipid variables (serum NEFA, HDL-cholesterol, non-HDLcholesterol, LDL particle size and VLDL-cholesterol), and glucose variables (fasting insulin, FPG and insulin sensitivity). The majority of the efficacy measures had not returned to baseline by the end of the 2-week washout period, and the drug was well tolerated over 12 weeks of treatment.

This study was conducted in individuals with hypertriglyceridaemia and abdominal obesity because the majority of such individuals are insulin resistant and these risk factors are associated with metabolic syndrome $[18,19]$. People with metabolic syndrome have a three-fold higher cardiovascular risk than those without metabolic syndrome [7-9], and are at high risk of developing type 2 diabetes [4-6].

The dyslipidaemia typical of insulin resistance associated with the metabolic syndrome and type 2 diabetes is highly atherogenic and is a major factor in the increased cardiovascular risk in this population. Such dyslipidaemia was seen in the patients recruited into SIR and is characterised by increased triglycerides and VLDL-cholesterol, reduced HDL-cholesterol and a shift in the size of LDL particles to small, dense LDL. The main drivers for many of these lipid abnormalities are hypertriglyceridaemia and insulin resistance [24] associated with NEFA overload. Typically, LDL particles become smaller and denser as the level of triglycerides rises. In this study, patients who received tesaglitazar had improvements in all of the lipid variables associated with diabetic dyslipidaemia, suggesting that tesaglitazar may have an important role to play in risk reduction.

The majority of type 2 diabetes patients have serum triglyceride levels in the range that results in the formation of small, dense LDL [13]. A particularly encouraging finding from this study was the highly significant change in the distribution of lipoprotein particle sizes towards the larger, less atherogenic pattern A. Among patients who received tesaglitazar $1.0 \mathrm{mg}, 79 \%$ of those who had pattern $\mathrm{B}$ at baseline had pattern $\mathrm{A}$ at the end of the 12-week treatment period. All patients in this group with pattern $\mathrm{A}$ at baseline retained the healthier pattern at the end of the study. In contrast, $25 \%$ of patients receiving placebo switched from pattern A to pattern B.

High non-HDL-cholesterol levels are an indicator of vascular risk [25] and the National Cholesterol Education Program Adult Treatment Panel III guidelines now spe- 
cifically recommend therapy in patients with hypertriglyceridaemia [17]. Changes in non-HDL-cholesterol (i.e. total cholesterol minus HDL-cholesterol) were evaluated in SIR, and patients treated with the two highest doses of tesaglitazar showed significant reductions in these levels.

Tesaglitazar is a dual-acting agonist of $\operatorname{PPAR} \alpha$ and PPAR $\gamma$. This combined activity should address the underlying abnormalities in both lipid and glucose handling associated with a wide range of related conditions, including insulin resistance, metabolic syndrome and type 2 diabetes. In the insulin-resistant population examined here, tesaglitazar had a significant antihyperglycaemic effect. Although the patients were not hyperglycaemic at baseline, they did have fasting glucose levels in the upper part of the normal reference range: mean fasting glucose concentrations ranged from 5.65 to $5.75 \mathrm{mmol} / \mathrm{l}$ at baseline. The fasting plasma glucose concentration currently accepted as a criterion for the diagnosis of diabetes is $7.0 \mathrm{mmol} / \mathrm{l}$, and individuals with concentrations of 6.1 to $6.9 \mathrm{mmol} / \mathrm{l}$ are considered to have impaired fasting glucose [26].

It might be expected that a glucose-lowering effect would be accompanied by a lowering of $\mathrm{HbA}_{1} \mathrm{c}$. However, the patients in this study were not hyperglycaemic and, as expected, had $\mathrm{HbA}_{1} \mathrm{c}$ levels in the normal reference range. Any reductions would be expected to be small and unlikely to be seen above random variations. $\mathrm{HbA}_{1} \mathrm{c}$ levels ranged from $5.65 \%$ to $5.79 \%$ at baseline and from 5.81 to $5.90 \%$ after 12 weeks. (The normal reference range is 4.0 to $6.0 \%$.) Furthermore, the duration of treatment was not sufficient for full appreciation of the effects on $\mathrm{HbA}_{1} \mathrm{c}$.

The development of clinical type 2 diabetes is almost always preceded by hyperinsulinaemia, and it has been suggested that hyperinsulinaemia is one of the pathological features responsible for the increased cardiovascular risk [27-29]. All the measures of insulin resistance investigated (fasting plasma insulin concentration, the HOMA index, and the product of fasting plasma insulin and fasting plasma NEFA) showed that tesaglitazar increased insulin sensitivity. For example, tesaglitazar reduced the elevated insulin levels in this study population, and the effect was statistically significant even at a dose of $0.25 \mathrm{mg}$ tesaglitazar per day.

There was a small increase in body weight in patients who received tesaglitazar. The potential for the drug to induce weight gain should be evaluated in further longerterm clinical trials. However, it is possible that the PPAR $\alpha$ agonist component of tesaglitazar acts to counter weight gain induced by stimulation of PPAR $\gamma$, an effect that may result from PPAR $\alpha$-induced lipid oxidation [30]. Similar to results reported for PPAR $\alpha$ agonists, such as fenofibrate, an initial dose-dependent increase in serum creatinine was observed that was reversible after the end of the study period [31]. Despite these increases, the absolute values remained within the normal ranges for adult men and women (70-114 $\mu \mathrm{mol} / 1$ for adult men; $53-88 \mu \mathrm{mol} / 1$ for adult women).

Insulin resistance is believed to be the core defect of a cluster of biochemical abnormalities: hyperinsulinaemia, hyperglycaemia, hypertriglyceridaemia, low-HDL-cholesterol and increased numbers of small, dense LDL particles and hypertension. This study has shown that tesaglitazar dose-dependently improves the abnormalities in lipid and glucose metabolism in patients with insulin resistance and dyslipidaemia. Because such insulin-resistant individuals are likely to develop type 2 diabetes [18-20], tesaglitazar may also have the potential to prevent vascular complications and delay progression to diabetes in this at-risk patient group. Further studies involving larger groups of patients should reveal the full effects of tesaglitazar.

Acknowledgements The authors would like to thank Dr Peter Öhman and all the investigators for their valuable contribution to the study. The authors also acknowledge the AstraZeneca publication team for editorial assistance.

Duality of interest:

B. Fagerberg and S. Edwards have received grants from AstraZeneca; H. Schuster has received an honorarium and grant from AstraZeneca; S. Tonstad has received grants and lecture/other fees from AstraZeneca; and S. Halldórsdóttir and I. Gause-Nilsson are employed by AstraZeneca.

\section{References}

1. Reaven GM (1988) Banting lecture 1988. Role of insulin resistance in human disease. Diabetes 37:1595-1607

2. Reaven GM (1995) Pathophysiology of insulin resistance in human disease. Physiol Rev 75:473-486

3. Haffner SM, Mykkanen L, Robbins D et al (1995) A preponderance of small dense LDL is associated with specific insulin, proinsulin and the components of the insulin resistance syndrome in non-diabetic subjects. Diabetologia 38:1328-1336

4. Hanson RL, Imperatore G, Bennett PH, Knowler WC (2002) Components of the 'metabolic syndrome' and incidence of type 2 diabetes. Diabetes 51:3120-3127

5. Lorenzo C, Okoloise M, Williams K, Stern MP, Haffner SM (2003) The metabolic syndrome as predictor of type 2 diabetes: the San Antonio Heart Study. Diabetes Care 26:3153-3159

6. Haffner SM, Stern MP, Hazuda HP, Mitchell BD, Patterson JK (1990) Cardiovascular risk factors in confirmed prediabetic individuals. Does the clock for coronary heart disease start ticking before the onset of clinical diabetes? JAMA 263:28932898

7. Isomaa B, Almgren P, Tuomi T et al (2001) Cardiovascular morbidity and mortality associated with the metabolic syndrome. Diabetes Care 24:683-689

8. Wilson PW, Kannel WB, Silbershatz H, D'Agostino RB (1999) Clustering of metabolic factors and coronary heart disease. Arch Intern Med 159:1104-1109

9. Sattar N, Gaw A, Scherbakova O et al (2003) Metabolic syndrome with and without C-reactive protein as a predictor of coronary heart disease and diabetes in the West of Scotland Coronary Prevention Study. Circulation 108:414-419

10. Lakka HM, Laaksonen DE, Lakka TA et al (2002) The metabolic syndrome and total and cardiovascular disease mortality in middle-aged men. JAMA 288:2709-2716

11. Hsia J, Bittner V, Tripputi M, Howard BV (2003) Metabolic syndrome and coronary angiographic disease progression: the women's angiographic vitamin \& estrogen trial. Am Heart J $146: 439-445$

12. McGarry JD (1992) What if Minkowski had been ageusic? An alternative angle on diabetes. Science 258:766-770

13. Taskinen MR (2002) Diabetic dyslipidemia. Atheroscler Suppl $3: 47-51$ 
14. Ljung B, Bamberg K, Dahllof B et al (2002) AZ 242, a novel PPARalpha/gamma agonist with beneficial effects on insulin resistance and carbohydrate and lipid metabolism in ob/ob mice and obese zucker rats. J Lipid Res 43:1855-1863

15. Ericsson H, Hamren B, Bergstrand S et al (2004) Pharmacokinetics and metabolism of tesaglitazar, a novel dual-acting peroxisome proliferator-activated receptor alpha/gamma agonist, after a single oral and intravenous dose in humans. Drug Metab Dispos 32:923-929

16. Alberti KG, Zimmet PZ (1998) Definition, diagnosis and classification of diabetes mellitus and its complications. Part 1: diagnosis and classification of diabetes mellitus provisional report of a WHO consultation. Diabet Med 15:539-553

17. NCEP Expert Panel (2002) Third report of the national cholesterol education program (NCEP) expert panel on detection, evaluation, and treatment of high blood cholesterol in adults (adult treatment panel III) final report. Circulation 106:31433421

18. Karter AJ, Mayer-Davis EJ, Selby JV et al (1996) Insulin sensitivity and abdominal obesity in African-American, Hispanic, and non-Hispanic white men and women. The insulin resistance and Atherosclerosis Study. Diabetes 45:1547-1555

19. Laaksonen DE, Lakka HM, Niskanen LK, Kaplan GA, Salonen JT, Lakka TA (2002) Metabolic syndrome and development of diabetes mellitus: application and validation of recently suggested definitions of the metabolic syndrome in a prospective cohort study. Am J Epidemiol 156:1070-1077

20. McAuley KA, Williams SM, Mann JI et al (2001) Diagnosing insulin resistance in the general population. Diabetes Care 24: 460-464

21. Otvos JD (1997) Measurement of lipoprotein subclass profile by nuclear magnetic resonance. In: Rifai $N$, Warnick $G$, Dominiczak M (eds) Handbook of lipoprotein testing. AACC Press, Washington DC, pp 329-333
22. Matthews DR, Hosker JP, Rudenski AS, Naylor BA, Treacher DF, Turner RC (1985) Homeostasis model assessment: insulin resistance and beta-cell function from fasting plasma glucose and insulin concentrations in man. Diabetologia 28:412-419

23. World Health Organization (1997) WHO adverse drug reaction terminology (WHOART). WHO Collaborating Centre for International Drug Monitoring, Uppsala, Sweden

24. Hulthe J, Bokemark L, Wikstrand J, Fagerberg B (2000) The metabolic syndrome, LDL particle size, and atherosclerosis: the atherosclerosis and insulin resistance (AIR) study. Arterioscler Thromb Vasc Biol 20:2140-2147

25. Wagner AM, Perez A, Zapico E, Ordonez-Llanos J (2003) Non-HDL cholesterol and apolipoprotein B in the dyslipidemic classification of type 2 diabetic patients. Diabetes Care 26: 2048-2051

26. Anonymous (2003) Report of the expert committee on the diagnosis and classification of diabetes mellitus. Diabetes Care 26(Suppl 1):S5-S20

27. Beckman JA, Creager MA, Libby P (2002) Diabetes and atherosclerosis: epidemiology, pathophysiology, and management. JAMA 287:2570-2581

28. Stout RW (1987) Insulin and atheroma-an update. Lancet 1: 1077-1079

29. Haffner SM, Mykkanen L, Festa A, Burke JP, Stern MP (2000) Insulin-resistant prediabetic subjects have more atherogenic risk factors than insulin-sensitive prediabetic subjects: implications for preventing coronary heart disease during the prediabetic state. Circulation 101:975-980

30. Larsen PJ, Jensen PB, Sorensen RV et al (2003) Differential influences of peroxisome proliferator-activated receptors gamma and alpha on food intake and energy homeostasis. Diabetes 52:2249-2259

31. Hottelart C, El Esper N, Rose F, Achard JM, Fournier A (2002) Fenofibrate increases creatininemia by increasing metabolic production of creatinine. Nephron 92:536-541 